\title{
A system for intensity modulated dose plan verification based on an experimental pencil beam kernel obtained by deconvolution
}

\author{
Juan Diego Azcona ${ }^{a)}$ \\ Department of Oncology, Division of Radiation Physics, Clinica Universitaria, Universidad de Navarra, \\ Avda. Pío XII, 36, 31008 Pamplona, Navarra, Spain \\ Javier Burguete \\ Department of Physics and Applied Mathematics, Facultad de Ciencias, Universidad de Navarra, \\ C/ Irunlarrea s/n, 31008 Pamplona, Navarra, Spain
}

(Received 5 March 2007; revised 17 October 2007; accepted for publication 19 October 2007; published 21 December 2007)

\begin{abstract}
The number of intensity modulated radiation therapy (IMRT) procedures is continuously growing worldwide and it is necessary to develop tools for patient specific quality assurance (QA) that avoid using machine time that could be employed in treating additional patients. One way of achieving this goal is to perform a multileaf collimator quality assurance periodically in the linear accelerator and check the treatment planning system (TPS) calculation by employing an independent calculation system. Within the work frame of the pencil beam kernel approach, a new system was developed for obtaining an experimental kernel. This new technique is based on a deconvolution procedure using the Hankel transform. The resulting kernel is obtained in a way completely independent of those employed in commercial treatment planning systems, usually calculated by Monte Carlo simulations. Also provided are comparisons between calculated and measured doses with radiographic film, linear array of diodes, and ionization chamber. Measurements taken in polystyrene and water for clinical IMRT plans demonstrate that this method can calculate IMRT dose distributions, as well as treatment times, with great accuracy. Apart from other applications, it can be used as a double-check algorithm for IMRT QA. (C) 2008 American Association of Physicists in Medicine.
\end{abstract} [DOI: $10.1118 / 1.2815359$ ]

Key words: deconvolution, Hankel transform, intensity modulation, IMRT treatment plan verification, pencil beam kernel

\section{INTRODUCTION}

It is common practice in radiotherapy to double-check the treatment planning system (TPS) calculations by an independent system, usually by hand calculations based on tissue phantom ratio (TPR) or percent depth dose (PDD) tables. In intensity modulated radiation therapy (IMRT) treatments this simple verification is not possible, since the dose inside the radiation field can vary abruptly between adjacent points. The radiation field can be viewed as composed of a number of beamlets, each one with a different fluence level. Furthermore, dose inside the field can have an important contribution from indirect radiation (scattered or transmitted through the multileaf collimator and jaws). An additional effect is that several penumbras due to different segments are added together inside the treatment field. These facts make the calculation of the absorbed dose in a medium quite complicated.

The accuracy of the TPS calculations in IMRT dose plans has frequently been ensured by measuring the absorbed dose of the plan on a phantom. Homogeneous phantoms have been widely used, in combination with radiographic film and ionization chambers. Although this practice gives a high degree of confidence in the treatment delivered to a patient, it has the main disadvantage of employing machine time that could be otherwise used to treat more patients. This is an inconvenience for implementing large IMRT programs, as well as for performing IMRT treatments in hospitals with a large number of patients.

A solution to this problem is to develop an algorithm for independent calculations. This double-check should be completed by an exhaustive and frequent QA of the multileaf collimator (i.e., a weekly QA). Guidelines for setting an IMRT program ${ }^{1-3}$ should be followed and it is the radiation physicist's responsibility to decide whether or not to substitute the measurements by independent calculations, when to do so, and whether the substitution should be total or partial.

Formalisms for independent calculations in IMRT have been developed by several authors. ${ }^{4-8}$ All of them perform their calculations in water. The article by Kung et al. ${ }^{4}$ was the first model for independent calculations of monitor units in IMRT treatment plans. They decomposed virtually the modulated field in circular sectors and applied a TPR algorithm for calculating the dose. Xing et al..$^{5}$ showed a quite general formalism. They modeled the direct fluence for each beamlet and then calculated the absorbed dose due to each beamlet. Their formalism can be used with a variety of dose calculation algorithms. Watanabe ${ }^{6}$ used a pencil beam algorithm for the calculation of the absorbed dose. The pencil beam kernel employed is described in the article by Ceberg et $a l .{ }^{9}$ and was convolved with the direct fluence to obtain the dose. These three articles describe algorithms for calcu- 
lating the dose at single points. Yang et $\mathrm{l}^{7}$ included the head scattered and transmitted radiation in the fluence modeling and extended the formalism by Xing et al. ${ }^{5}$ to calculate 2D dose distributions. They used the three source algorithm ${ }^{10}$ for modeling the scattered dose. This model involves a Monte Carlo simulation. Chui et al. ${ }^{8}$ also calculate dose distributions. They employ Monte Carlo calculations to generate the pencil beam kernels used.

A number of different algorithms can be used, in principle, to calculate the dose. Pencil beam kernels can be suitable for an independent verification algorithm that calculates dose in water, since they keep the accuracy of a 3D convolution algorithm in a homogeneous medium. Another important advantage of this algorithm is that the dose is calculated from the characterization of the fluence, and the fluence can easily be reconstructed from the file generated by the TPS and used by the linear accelerator to deliver the treatment plan. The well-known formula for the dose calculation using the pencil beam approach is

$$
\begin{aligned}
D\left(x, y, z_{0}\right) & =\iint_{S} \Phi\left(x^{\prime}, y^{\prime}\right) k\left(x-x^{\prime}, y-y^{\prime}, z_{0}\right) d x^{\prime} d y^{\prime} \\
& =\Phi(x, y) \otimes k(x, y),
\end{aligned}
$$

where $D$ denotes the absorbed dose, $\Phi$ is the fluence at the entrance surface $S$ of the medium, and $k$ is the pencil beam kernel. The kernel $k$ is polyenergetic (averaged for the energy spectrum considered). In the calculation of Eq. (1) we consider the section of the kernel $k$ at a fixed depth $z_{0}$. Then, the term $k\left(x-x^{\prime}, y-y^{\prime}, z_{0}\right)$ is actually a planar kernel, valid for calculating the dose distribution at that depth. Equation (1) represents a $2 \mathrm{D}$ convolution that can be calculated in a homogeneous medium once the spatial invariance of the kernel is assumed. ${ }^{11}$ Furthermore, the kernel tilt due to beam divergence is not considered, which is equivalent to considering a parallel beam.

The central point is the characterization of the pencil beam kernel. There are a number of different ways for achieving this goal. Numerical Monte Carlo simulation, empirical fitting to analytical expressions, and deconvolution are three alternatives for obtaining pencil beam kernels. In the following, we provide references of how these different approaches have been investigated and implemented.

Ahnesjo $^{12}$ calculated by Monte Carlo the monoenergetic pencil beam kernels and fitted them to an analytical expression to characterize the polyenergetic kernel. Mohan and Chui ${ }^{11}$ calculated by Monte Carlo the polyenergetic kernel, taking into account the energy spectrum of the linear accelerator. Bortfeld et al. ${ }^{13}$ fitted an analytical expression for the pencil beam kernel according to dose distributions calculated by convolution-superposition using Monte Carlo generated point dose kernels. Bourland and Chaney ${ }^{14}$ developed the finite-size pencil beam model where the pencil beam was calculated by convolving the photon fluence with Monte Carlo point dose kernels along the depth coordinate. Their model was originally developed for ${ }^{60} \mathrm{Co}$ photons.

A different approach is to characterize a pencil beam kernel from measurements instead of from Monte Carlo simu- lations. This has been carried out by two different groups. ${ }^{9,15-19}$ They fitted the polyenergetic pencil beam kernel to an analytical expression according to experimental data usually measured during the commissioning procedure.

The approach we are considering consists of obtaining an experimental pencil beam kernel by deconvolution. The most relevant feature of this approach is that the kernel is measured, instead of being simulated or fitted using experimental data. This way was initially explored by Chui and Mohan ${ }^{20}$ and no further research work has been performed since then. As they state in their work, pencil beam kernels thus obtained take into account the beam spectrum hardening with depth. This is an important feature in favor of this experimental technique.

Since dose in a homogeneous medium is the convolution of the incident fluence and a pencil beam kernel, as noted in Eq. (1), one can obtain the kernel from a measured dose distribution, using the Fourier (or another suitable) transform. The fundamental property that the employed transform should have is to convert convolutions in the real space to products in the transformed space. Taking the generic transform GT on Eq. (1),

$$
\mathrm{GT}(\Phi \otimes k)=\mathrm{GT}(\Phi) \times \mathrm{GT}(k)=\mathrm{GT}(D),
$$

where the symbol $\otimes$ represents the convolution operation. Then, the kernel can be obtained as

$$
k=\mathrm{GT}^{-1}\left(\frac{\mathrm{GT}(D)}{\mathrm{GT}(\Phi)}\right) \text {. }
$$

The procedure of Chui and Mohan has been developed for radiation fields with some modulation of fluences, such as blocked or wedged fields. ${ }^{20}$ Intensity modulated fields have much more modulation, and a new deconvolution technique is needed for these cases. The procedure of Chui and Mohan relays on 1D dose profiles assuming that the kernel can be decomposed in a product where each factor carries the dependence of the kernel with each of the spatial components $x$ and $y$. This point will be further discussed in the next section.

In the following, we describe a method for an independent calculation of IMRT dose distributions and treatment times based on an experimental pencil beam kernel obtained by 2D deconvolution. The mathematical procedure for the derivation of the kernel is described in detail, as well as the measurement conditions for the required radiation fields. Finally, we present some comparisons between calculations and measurements with radiographic film, linear array of diodes, and ionization chamber in clinical IMRT fields.

\section{THEORY}

\section{II.A. Formalism for the independent calculation of absorbed dose}

We employ the pencil beam approach, where the absorbed dose for a modulated field can be calculated according to Eq. (1). The incident fluence $\Phi$ is the sum of a primary $\Phi_{\text {prim }}$ 
plus a head scattered $\Phi_{\mathrm{hs}}$ component. Assuming a primary point source, the incident fluence can be expressed as

$$
\Phi=\Phi_{\text {mod }}^{\prime} \otimes s_{e},
$$

where $\Phi_{\text {mod }}^{\prime}$ denotes the modulated primary fluence coming from a point source. The total fluence can be expressed as the convolution of $\Phi_{\text {mod }}^{\prime}$ with a source distribution $s_{e}$. The term $s_{e}$ represents the finite extent of the source, ${ }^{11}$ and consequently how the ideal point source is spread in $2 \mathrm{D}$ (the corresponding units are $1 / \mathrm{m}^{2}$ ). It contains a component that takes into account the finite extent of the primary source as well as a second component that accounts for the head scatter, which comes mainly from the flattening filter.

The terms fluence $(\Phi)$ and modulated primary fluence coming from a point source $\left(\Phi_{\text {mod }}^{\prime}\right)$ used in Eqs. (1) and (4) have units of $\mathrm{J} / \mathrm{m}^{2}$ since they actually represent energy fluence. The kernel $k$ used in Eq. (1) has units of $\mathrm{kg}^{-1}$, so that the absorbed dose has units of Gy.

Regarding the fluence characterization, we make use of the concept of modulation index for each beamlet. ${ }^{5} \mathrm{~A}$ beamlet is the smallest area in which a modulated field can be virtually decomposed with homogeneous modulation. Let us consider a modulated field as composed of $K$ segments and $M$ beamlets. Each segment $\kappa$ has an irradiation time of $\mathrm{MU}_{\kappa}$. If $\mathrm{MU}$ is the total irradiation time, $f_{\kappa}$ is defined as the ratio $\mathrm{MU}_{\kappa} / \mathrm{MU}$. Let $\alpha$ be the fraction of transmitted fluence through the MLC with respect to the fluence in open field. According to Xing et al. ${ }^{5}$ the modulation index $C_{m}$ for beamlet $m$ is defined as

$$
C_{m}=\sum_{\kappa}^{K}\left[\delta_{m, A_{\kappa}}+\alpha\left(1-\delta_{m, A_{\kappa}}\right)\right] f_{\kappa},
$$

where $A_{\kappa}$ represents the shape for segment $\kappa$. With this notation, $\delta_{m, A_{\kappa}}=1$ if $m \in A_{\kappa}$, and $\delta_{m, A_{\kappa}}=0$ if $m \notin A_{\kappa}$. The modulation index describes the fluence in a beamlet $m$. Note that $C_{m} \leq 1$ and for open field $C_{m}=1$.

Second-order effects such as tongue and groove and interleaf transmission are included in the fluence reconstruction according to the formalism described by Chui et al. ${ }^{8} \mathrm{~A}$ slight correction is introduced only in the points affected by these effects. The modulation index $C_{m}$ is replaced by $C_{m}^{\text {corr }}$ for the fluence reconstruction.

We characterize the modulated primary fluence $\Phi_{\text {mod }}^{\prime}$ at a point $\left(x^{\prime}, y^{\prime}\right)$ inside a beamlet $m$ as follows:

$$
\Phi_{\text {mod }}^{\prime}=C_{m}^{\mathrm{corr}} \text {. }
$$

In this way, the fluence $\Phi_{\text {mod }}^{\prime}$ is a relative fluence referenced to the maximum value obtained with an open field with the total irradiation time MU.

In the fluence map reconstruction we have chosen a space between adjacent positions of $1 \mathrm{~mm}$. As the maximum field size is $40 \times 40 \mathrm{~cm}^{2}$ in our linear accelerator, the matrices that contain the fluence maps have $400 \times 400$ points. The fluence reconstruction was done based on the leaf positioning information contained in the file generated by the TPS and sent to the linear accelerator for delivering the treatment.
The absorbed dose is calculated according to the following equation:

$$
\begin{aligned}
D\left(x, y, z_{0}\right)= & D_{\mathrm{MU}}^{\mathrm{ref}} \mathrm{MU} \operatorname{OAR}(x, y)\left[\Phi_{\bmod }^{\prime} \otimes w\right] \\
= & D_{\mathrm{MU}}^{\mathrm{ref}} \operatorname{MU} \operatorname{OAR}(x, y) \iint_{S} \Phi_{\bmod }^{\prime}\left(x^{\prime}, y^{\prime}\right) \\
& \times w\left(x-x^{\prime}, y-y^{\prime}, z_{0}\right) d x^{\prime} d y^{\prime} .
\end{aligned}
$$

Since the fluence in Eq. (7) is a modulated primary fluence, $w=s_{e} \otimes k$ is the convolution of a phantom scatter kernel $k$ [the kernel in Eq. (1)] with $s_{e}$, which includes the characterization of the finite extent primary source and the head scatter source. The kernel $w$ is the quantity that will be obtained by deconvolution. $D_{\mathrm{MU}}^{\text {ref }}$ is the reference dose rate (dose per monitor unit). MU denotes the total number of monitor units for the modulated field. The OAR is the ratio of the dose at a point located at $(x, y)$ coordinates far from the central axis and at a given depth and the dose at a point at the same depth on the central axis $(0,0)$, defined in an open field. With this term the deviations in a real open field from the dose distribution due to an ideal flat energy fluence are taken into account. ${ }^{8}$ The convolution results in a normalized dose of unity for the reference field at isocenter and this corresponds to $D_{\mathrm{MU}}^{\mathrm{ref}}$ cGy per monitor unit in our linear accelerator conditions. For calculating the dose at an off-axis point the additional multiplication by the OAR is required. The determination of $w$ allows an accurate calculation of the absorbed dose. Performing the calculation of the convolution integral and multiplying it by OAR and $D_{\mathrm{MU}}^{\mathrm{ref}}$ will lead us to the dose distribution, which contains the effects of the geometrical and physical penumbra.

In our procedure, we are calculating the dose due to a fluence map known at the entrance of the absorbing medium. The dose distributions will be calculated at $15 \mathrm{~cm}$ depth in water, assuming a flat entrance surface. The quantity OAR at this depth has been measured with a diode, taking a diagonal profile of the maximum available open field in water. The pencil beam kernels we use have been calculated from dose distributions measured with radiographic film at $15 \mathrm{~cm}$ equivalent depth in water. We have chosen this depth as being typical for IMRT patients. We assume that the calculation plane is located at $100 \mathrm{~cm}$ from the source (at the isocenter) and that the radiation beam is not divergent but parallel. We calculate separately each beam of a treatment plan in the described conditions.

For the calculation of the convolution of expression (7), the fast Fourier transform algorithm (FFT) in the MATLAB environment was employed. Once the kernel was obtained and stored, the time required to calculate the dose distributions for a seven field IMRT dose plan was less than a minute on a $3.06 \mathrm{GHz}$ Pentium 4 processor.

\section{II.B. Deconvolution procedure for obtaining the pencil beam kernel}

The research by Chui and Mohan ${ }^{20}$ used a 1D Fourier transform that leads to a 1D kernel. They assume that the 2D pencil beam kernel can be expressed as $k(x, y)=k_{1 \mathrm{D}}(x)$ 
$k_{1 \mathrm{D}}(y)$ and employ a step function to characterize the fluence on a square field. Each component describes the kernel behavior on each orthogonal direction. This approach allows the decoupling in the convolution product: the dose can be computed along a given direction (i.e., $x$ axis) under the assumption that the convolution along the other direction $(y$ axis) is a constant, which depends on the field extension in the $y$ axis (equal to one for infinite, smaller than one for finite fields). ${ }^{20}$ But strictly speaking, the kernel cannot be expressed generically according to the above expression, since it has cylindrical symmetry. Only in a few special cases is this separation possible (i.e., Gaussian kernels). For a kernel where the variables are nonseparable, the convolution along the $y$ direction will lead generically to a modulation in the other direction that will be $x$ dependent and will not be a constant.

Nevertheless, the kernel obtained with this method will approximate the dose calculated with the real kernel, provided that the field can be considered infinite in the $y$ direction. The main point is to define under which conditions this approximation will be valid. Let us suppose that the fluence varies in space with a typical scale $L_{\Phi}$ and the kernel has a spatial extension $L_{k}$ (i.e., the diameter that contains $90 \%$ of the kernel). Then, if the field is modulated in such a way that $L_{\Phi} \gg L_{k}$, the dose calculations will be similar, whereas if $L_{\Phi} \approx L_{k}$, the separation variable method gives erroneous dose calculations. As the typical kernel length is in the range of several millimeters, we should consider that this approach will be no longer valid for fluence modulations of the order of the centimeter, the typical beamlet side length in IMRT. In those fields, the real 2D behavior of the kernel becomes crucial.

For these reasons, we have developed a new method, based on the zeroth-order Hankel transform, also known as the Fourier-Bessel transform. The key feature is to obtain the kernel by $2 \mathrm{D}$ deconvolution using a dose distribution due to a relatively small circular field, $50 \mathrm{~mm}$ in diameter. The reason for using a circular field instead of a square one is mainly because of the revolution symmetry of the problem. Any other approach different from this work frame means making approximations. Furthermore, there are some methodological advantages. All points in the perimeter of the circular field equally contribute to the dose generation from the kernel. For a square field, only the areas near the corners give information about the $2 \mathrm{D}$ behavior of the kernel. In addition to this, it is possible to obtain the 2D kernel using a 1D transform. The cost for these goals is the use of the zeroth-order Hankel transform, where we can make use of the cylindrical symmetry of the problem.

The expression of the zeroth-order Hankel transform is analogous to the definition of the Fourier transform. In the Fourier transform, we express a function in terms of sines and cosines, whereas in the zeroth-order Hankel transform we express the function in terms of the Bessel function of first kind and zeroth order.

\section{II.C. The zeroth-order Hankel transform (Fourier- Bessel transform)}

Let the 2D Fourier transform of a function $f(x, y)$ (in Cartesian coordinates) be

$$
F(\mathbf{q})=F\left(q_{x}, q_{y}\right)=\int_{-\infty}^{\infty} \int_{-\infty}^{\infty} f(x, y) e^{-i\left(q_{x} x+q_{y} y\right)} d x d y .
$$

If $f(x, y)$ has cylindrical symmetry, we can express $f(x, y)$ $=f(r)$, and, as $x=r \cos \theta$ and $y=r \sin \theta$,

$$
F(\mathbf{q})=\int_{0}^{\infty} \int_{0}^{2 \pi} r f(r) e^{-i r\left(q_{x} \cos \theta+q_{y} \sin \theta\right)} d r d \theta .
$$

Let us now introduce the modulus $q$ as the independent variable in the transform: $q_{x}=q \cos \varphi, q_{y}=q \sin \varphi$. The transform can be written as

$$
F(q, \varphi)=\int_{0}^{\infty} \int_{0}^{2 \pi} r f(r) e^{-i r q(\cos \theta \cos \varphi+\sin \theta \sin \varphi)} d r d \theta .
$$

Since $\cos \theta \cos \varphi+\sin \theta \sin \varphi=\cos (\theta-\varphi)=\cos \theta^{\prime}$, we can calculate the angular integral using $\theta^{\prime}$. Then, we reach the following expression:

$$
\begin{aligned}
F\left(q, \theta^{\prime}\right) & =\int_{0}^{\infty} \int_{0}^{2 \pi} r f(r) e^{-i q r \cos \theta^{\prime}} d r d \theta^{\prime} \\
& =\int_{0}^{\infty} r f(r) J_{0}(q r) d r \\
& =F(q),
\end{aligned}
$$

where $J_{0}(q r)$ is the first kind zeroth-order Bessel function, defined as $J_{0}(q r)=\int_{0}^{2 \pi} e^{-i q r \cos \theta^{\prime}} d \theta^{\prime}$. The function $F\left(q, \theta^{\prime}\right)$ does not depend on $\theta^{\prime}$, but it has cylindrical symmetry, so we can just write $F(q)$. The zeroth-order Hankel transform in the continuous case keeps the fundamental property of the Fourier transform we need: convolutions are converted to products in the transformed space. ${ }^{21}$

Now we turn to the discrete zeroth-order Hankel transform, ${ }^{22-24}$ which is the form of this transform that we will use in fact. It is analogous to the discrete Fourier transform, which is actually used in many physical problems instead of the Fourier transform definition.

Let us note that the zeroth-order discrete Hankel transform is, in fact, a quasi-discrete transform. This means that the Bessel functions set forms a near-orthogonal base, instead of an orthogonal base, ${ }^{23,24}$ when a finite series of functions is taken. But, as the base is very close to be orthogonal strictu sensu, in practice the convolution property can be used. We have performed our own implementation of an algorithm for calculating the zeroth-order Hankel transform, based on the work by Lemoine ${ }^{22,23}$ and Yu et al. ${ }^{24}$ (see the Appendix). The number of zeros to calculate depends on the number of data in the original signal. In order to obtain the kernel by deconvolution using Hankel, we have used a number of data $N=400$. The accuracy for performing a pair of direct and back transforms was $10^{-15}[$ (signal $-H^{-1}(H($ signal $\left.))\right) /$ signal] $(H$ denotes Hankel transform). 


\section{MATERIAL AND METHODS}

IMRT treatments are performed at our Institution on Siemens Oncor and Mevatron Primus linear accelerators, both equipped with multileaf collimators. All film measurements presented in this work concerning the kernel derivation, as well as the comparison between calculated dose distributions and absolute values for absorbed dose with measurements, have been performed with radiographic Kodak XV film exposed in the Mevatron Primus. We shall separate this section in two parts: the first part deals with the kernel measurement method and the second one with the dose measurement conditions in IMRT clinical plans.

\section{III.A. Kernel measurement}

In order to apply the Fourier-Bessel transform method, a circular radiation field is needed. A lead collimator was manufactured that defines a $50 \mathrm{~mm}$ diameter beam. The collimator was positioned on the block tray of the linear accelerator, $56 \mathrm{~cm}$ from the target. Its thickness was $8 \mathrm{~cm}$. The hole had a cone shape with a lower diameter of $28 \mathrm{~mm}$ and an upper diameter of $24 \mathrm{~mm}$. The block sectional shape was square, $10 \times 10 \mathrm{~cm}^{2}$ size. The jaws positions for taking the films were $10 \times 10 \mathrm{~cm}^{2}$ (at isocenter).

The selected geometry for our measurements was source to film distance of $100 \mathrm{~cm}$ and depth of solid water (white polystyrene, RW3 Solid Phantom Type 29672, PTW Freiburg) of $15 \mathrm{~cm}$. Profiles in photon beams are equivalent to those measured in water. This depth was chosen as being typical for IMRT treatments.

Kernels for 6 and 15 MV energies were measured. For each one of the energies, several irradiations were performed, with a different number of monitor units: 20, 25, 40, $50,60,70,80,90$, and 100. Of course, all of these different irradiations should lead to the same kernel. This was done to find a good range of irradiation time where the kernel can be properly measured with the selected setup and radiographic film.

Special attention was paid to the film calibration procedure. The calibration table had 23 steps, in between 5 and 110 monitor units every 5 monitor units, plus a base+fog film. This procedure was done in order to reconstruct the dose in the penumbra region with great accuracy, which is crucial to obtain a reliable kernel. The films were developed and scanned. A very important characteristic the scanner should have is good resolution, both in the high dose and in the low dose area. The information contained in the latter is also essential for a good kernel derivation.

Once the films were developed, an averaged radial profile was taken from each of the films to apply the Fourier-Bessel transform. We employed 360 radial dose profiles, equally spaced in angle in steps of $1 \mathrm{deg}$. The circular $50 \mathrm{~mm}$ field profile used for deriving the pencil beam kernel was also divided by the OAR prior to apply the deconvolution technique. The OAR was measured at the depth at which the section of the pencil beam kernel was obtained, that is, 15 $\mathrm{cm}$. We also modeled the incident fluence as a step function with revolution symmetry. With these elements we applied the formalism previously described to obtain the pencil beam kernel $w$. We have developed our own computer program to calculate the Fourier-Bessel transform.

\section{III.B. Clinical IMRT measurements and comparison}

In this part we describe the procedure for measuring and comparing the IMRT doses with calculations done according to the pencil beam approach based on the kernel obtained by deconvolution. The calculation formalism is described above in Sec. II. We will provide dose profiles for IMRT fields measured with radiographic film in polystyrene and linear array of diodes in water, as well as absolute dose measurements at single points taken with the ionization chamber in polystyrene and transformed to absorbed dose in water. The measured and calculated profiles were normalized in a flat high dose area, where a single dose measurement was also performed with a $0.125 \mathrm{~cm}^{3}$ ionization chamber. These fields were designed and used for patient treatments. The same sequences of segments with the treatment times used for the patients were delivered to the water and polystyrene phantoms in order to get the measurements. The films employed were Kodak XV, inserted in white polystyrene slabs. They were irradiated at $100 \mathrm{~cm} \mathrm{SAD}$ and $15 \mathrm{~cm}$ depth in polystyrene. Measurements were taken for several IMRT fields with a number of segments, corresponding to a single fixed gantry position. The range of the number of segments was between 7 and 14. The range in the number of monitor units was between 9 and 18. All these fields were used clinically and were chosen as being typical of our treatment situations. Table I specifies the number and size of the segments for each field. The same film calibration schedule and recommendations described in the kernel measurement section were followed here, as well as the system and procedure used for scanning. Comparisons were established between the IMRT fields measured and the calculations performed with our kernels, both in relative profiles and in absolute dose values at single points. The aim of these comparisons is to verify that the experimental pencil beam kernels derived according to this procedure are adequate to calculate IMRT dose distributions with great accuracy. Furthermore, a series of single dose measurements with ionization chamber is taken on a profile and results are compared to absolute dose calculations.

TABLE I. Characterization of the number and size of the segments employed in the clinical IMRT fields.

\begin{tabular}{ccccc}
\hline \hline Case & Segments & $\begin{array}{c}\text { Minimum size } \\
\left(\mathrm{cm}^{2}\right)\end{array}$ & $\begin{array}{c}\text { Maximum size } \\
\left(\mathrm{cm}^{2}\right)\end{array}$ & $\begin{array}{c}\text { Mean size } \\
\left(\mathrm{cm}^{2}\right)\end{array}$ \\
\hline 1 & 14 & 15.8 & 60.1 & 38.8 \\
2 & 10 & 23.1 & 100.4 & 70.4 \\
3 & 10 & 25.5 & 98 & 67.8 \\
4 & 7 & 24.2 & 224 & 113.3 \\
5 & 9 & 28.1 & 54.5 & 41.1 \\
6 & 8 & 32 & 164.5 & 80.1 \\
7 & 10 & 13.3 & 48.1 & 35.6 \\
\hline \hline
\end{tabular}


A final comparison is provided between calculated $\left(D_{c}\right)$ and measured with radiographic films $\left(D_{m}\right)$ doses. The criterion for comparison is the gamma index as defined in the work by Low et al.:25

$$
\begin{aligned}
\gamma\left(\mathbf{r}_{m}\right)= & \min \left\{\sqrt{\frac{\left(\left|\mathbf{r}_{c}-\mathbf{r}_{m}\right|\right)^{2}}{\Delta d_{M}^{2}}+\frac{\left(D_{c}\left(\mathbf{r}_{c}\right)-D_{m}\left(\mathbf{r}_{m}\right)\right)^{2}}{\Delta D_{M}^{2}}}\right\} \\
& \forall \mathbf{r}_{c},
\end{aligned}
$$

where $\mathbf{r}_{m}$ denotes the position of the measured point whose dose is going to be compared, and $\mathbf{r}_{c}$ is the position of the series of points surrounding $\mathbf{r}_{m}$ whose calculated dose and distance are the matter of comparison. $\Delta d_{M}$ is the acceptance criterion for distance and $\Delta D_{M}$ is the acceptance criterion for dose. Then, the criterion for the gamma index is
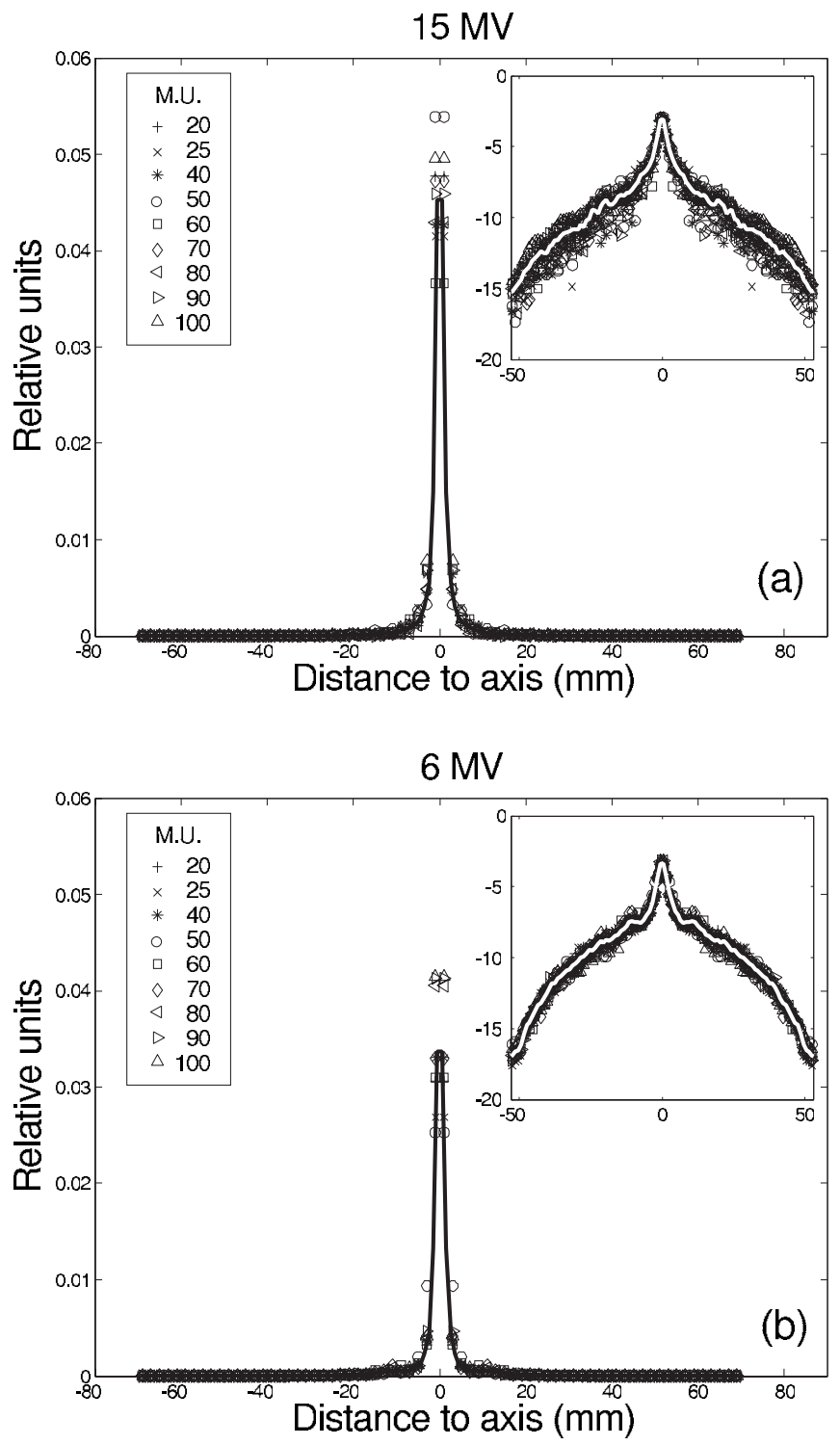

FIG. 1. Kernels obtained for the different irradiation times specified in the text. The final kernel is the average of all (black solid curve). The inset represents the same plot in logarithmic view, with the final kernel in white.

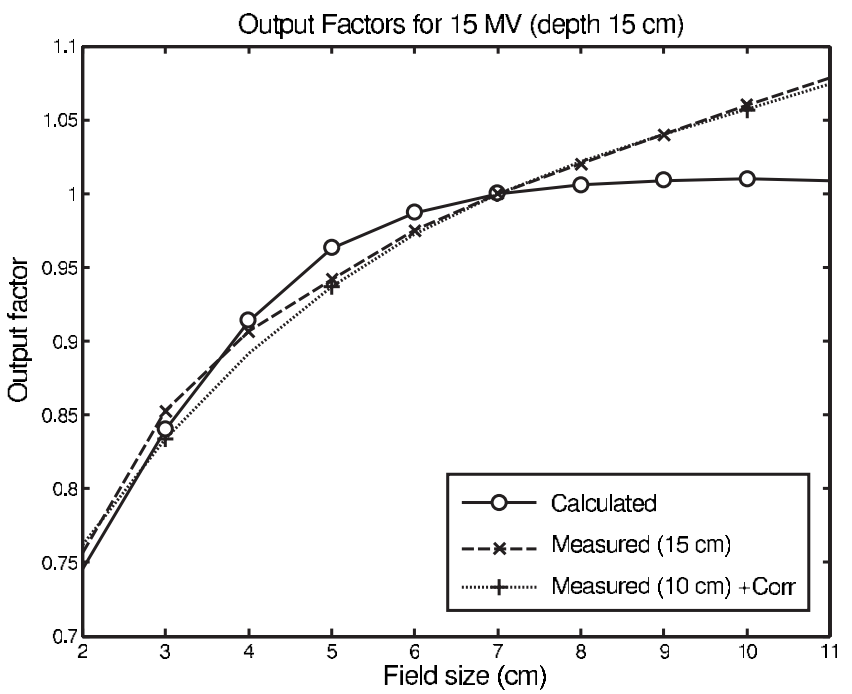

FIG. 2. Reconstructed output factors calculated with the kernel obtained by deconvolution and compared with the measured ones in a $15 \mathrm{MV}$ photon beam from a Siemens linear accelerator. Two measured series are presented: one of them presents measured values at $15 \mathrm{~cm}$ and the other presents values originally measured at $10 \mathrm{~cm}$ depth and corrected to the value at $15 \mathrm{~cm}$ by a ratio of TPR. Values are normalized with respect to a $7 \times 7 \mathrm{~cm}^{2}$ field. The kernel was derived in such conditions that scatter coming further than $7 \mathrm{~cm}$ distance was ignored (see text). The error associated with the measurements is $1 \%$.

$\gamma\left(\mathbf{r}_{m}\right) \leq 1$, the calculation passes (criterion fulfilled),

$\gamma\left(\mathbf{r}_{m}\right)>1$, the calculation fails (criterion not fulfilled).

The tolerances employed by us are $\Delta D_{M}=3 \%$ in dose and $\Delta d_{M}=3 \mathrm{~mm}$ in distance. Here the measured dose values used for the comparison are absolute ones. Histograms with the gamma index values for each dose matrix are provided, where a gamma value equal to or less than 1 represents good agreement between the compared dose matrices.

The data provided are for IMRT dose plans performed with $15 \mathrm{MV}$ photons, which is the energy usually employed in our Institution for IMRT procedures. We treated a few patients with $6 \mathrm{MV}$ photons, and calculations for this energy with our algorithm as well as comparisons with experimental measurements will be the subject of future development.

\section{RESULTS AND DISCUSSION}

\section{IV.A. Kernels obtained by Fourier-Bessel deconvolution}

Figures 1(a) and 1(b) show the kernels obtained for the 15 and $6 \mathrm{MV}$ photons, respectively, for the different treatment times employed, as summarized in Sec. III A. The continuous line represents the final kernel, after taking the average for all the kernels represented in the figure (on logarithmic scale in the inset). These averaged kernels correspond to the Mevatron Primus 15 and 6 MV photon beams.

The best approach to reduce noise is to measure a series of several films with identical experimental conditions. However, in order to test the influence of noise and saturation level, we chose a different approach. A series of films was 

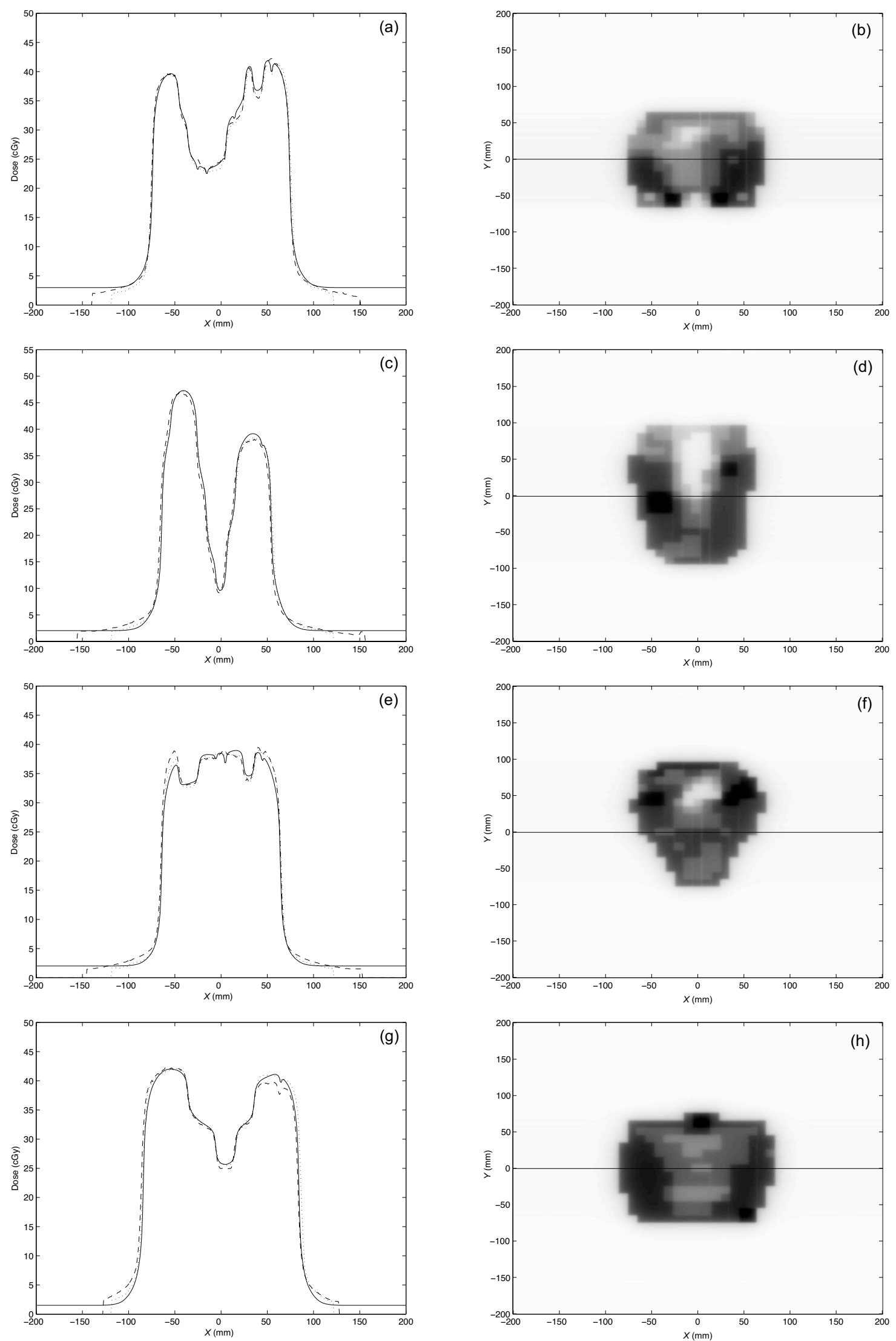

Fig. 3. Comparison between calculations (solid), array measurements (dotted), and film measurements (dashed) for several IMRT fields used in clinical cases [cases: (a) 1, (c) 2, (e) 3, (g) 4, (i) 5, (k) 6, and (m) 7]. Dose values are provided and renormalized to the absorbed dose measured with the ionization chamber in a high dose, low gradient area (points in Table I). The profile direction is indicated over the dose map [cases: (b) 1, (d) 2, (f) 3, (h) 4, (j) 5, (1) 6, and (n) 7]. The photon energy is $15 \mathrm{MV}$. 

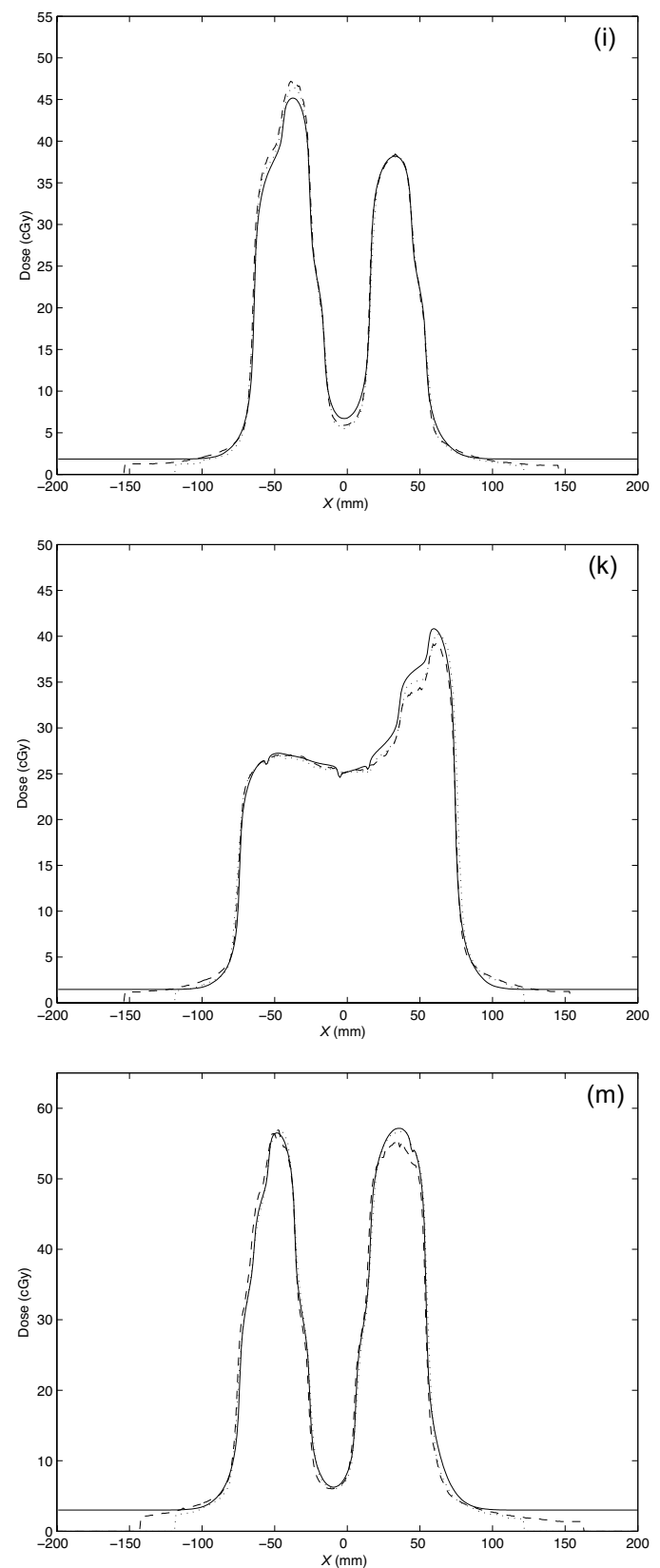
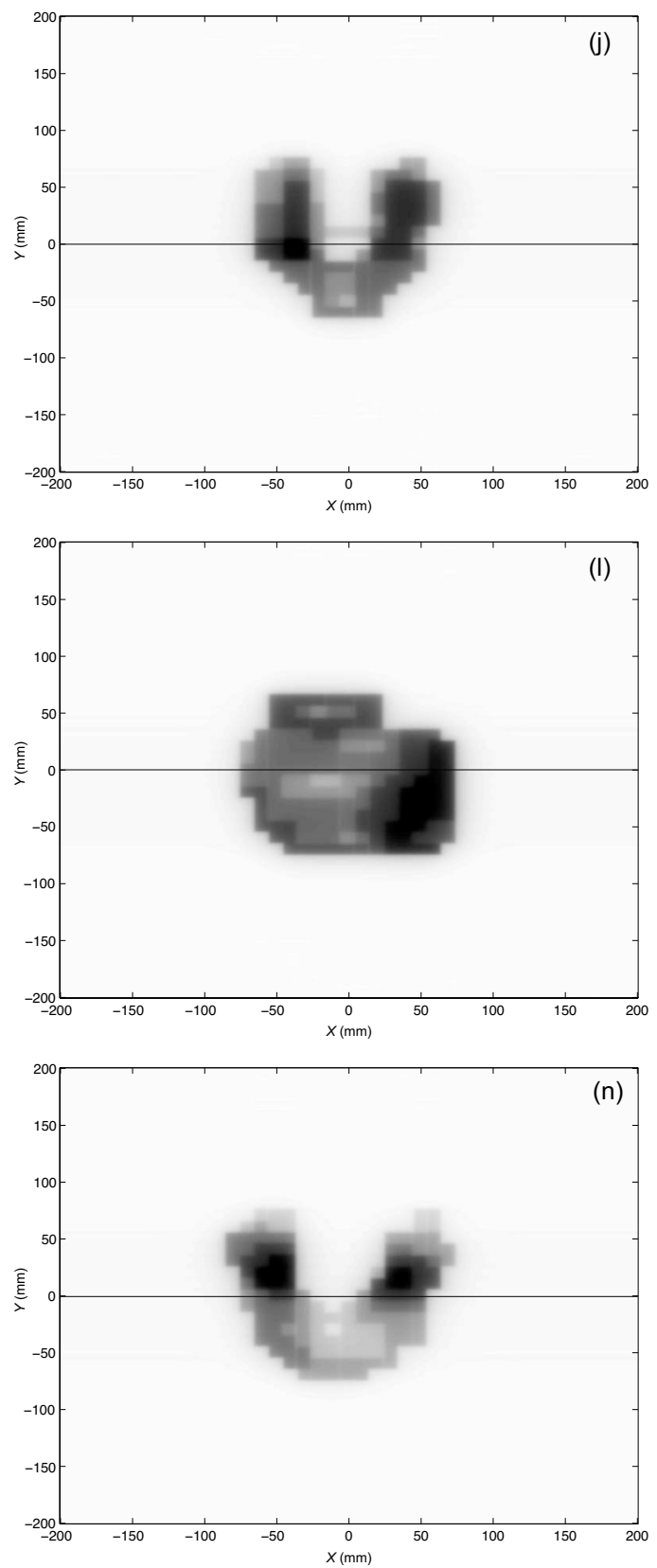

Fig. 3. (Continued).

exposed with different irradiation times. This practice allowed us to recommend an irradiation time as high as possible (in our case, the optimal number of monitor units was 70-90), taking care not to exceed the saturation level for the film. Finally, we averaged the resulting kernels for all the films to obtain the final kernel employed in our calculations.

The averaged kernel obtained for $15 \mathrm{MV}$ photons represented in Fig. 1(a) was used for our calculations in all the clinical IMRT cases shown in the next subsection. The head scatter contribution is overestimated since the portion of flattening filter viewed from a point in the isocenter plane through a circular hole located at $56 \mathrm{~cm}$ from the source will always be bigger than the portion viewed from the same point through a circular hole that conforms the same field at isocenter but located at the MLC position, closer to the source.

We reconstructed the output factors table with our derived pencil beam kernel (Fig. 2). The calculated output factors coincide properly (with a difference of less than $3 \%$ ) below field sizes of approximately $8 \mathrm{~cm}$. The curve shows a "saturation" effect above the $10 \mathrm{~cm}$ square field. This is directly related to the procedure for obtaining the pencil beam kernel. Because of the experimental procedure employed for the deconvolution, we only had access to areas of $15 \times 15 \mathrm{~cm}^{2}$, so the maximum radius with information useful for the transform was around $7 \mathrm{~cm}$. Thus, information from higher radii 
TABLE II. Comparison between absolute dose calculations and measurements in high dose, low gradient area (15 MV photons). In those points the profiles have been normalized. These profiles are taken at $y=0$, and the off-axis positions correspond to the $x$ coordinate.

\begin{tabular}{ccccc}
\hline \hline Case & $\begin{array}{c}\text { Off axis position } \\
(\mathrm{mm})\end{array}$ & $\begin{array}{c}\text { Calculated dose } \\
(\mathrm{cGy})\end{array}$ & $\begin{array}{c}\text { Measured dose } \\
(\mathrm{cGy})\end{array}$ & $\begin{array}{c}\text { Difference } \\
(\%)\end{array}$ \\
\hline 1 & -60 & 38.4 & 39.2 & -2.0 \\
2 & -45 & 45.7 & 47.0 & -2.8 \\
3 & 0 & 38.8 & 38.5 & 0.8 \\
4 & -50 & 41.9 & 43.0 & -2.6 \\
5 & +35 & 38.7 & 38.1 & 1.6 \\
6 & 0 & 24.4 & 25.2 & -3.2 \\
7 & -50 & 57.4 & 56.4 & 1.8 \\
\hline \hline
\end{tabular}

was lost. This is a limitation due to the current implementation of our method and a new design of the measurement setup is matter of future refinement. The pencil beam kernel will work well for fluence maps with a high degree of modulation, since the effect of the kernel fall is dominant in those areas, whereas the kernel tail has more importance in relatively big and homogeneous fields, as can be justified with the reconstructed output factor curve.

Let us note, finally, that the resulting kernel cannot be adjusted to an exponential function nor a Gaussian one. In the logarithmic view in Fig. 1, one can conclude that there are three linear areas, so a sum of at least three exponentials on radius could, in principle, represent accurately the kernel.

The method presented here is not depth dependent and should work for all depths. Nevertheless, the results presented correspond to $z_{0}=15 \mathrm{~cm}$.

\section{IV.B. Clinical IMRT plans calculated and measured}

In this subsection a comparison will be provided between measurements of clinical IMRT treatment plans and calculations according to the algorithm previously described, using an experimental pencil beam kernel for $15 \mathrm{MV}$ photons obtained with Fourier-Bessel deconvolution. Figure 3 shows the comparison between the results of the calculation with the $15 \mathrm{MV}$ kernel and the measurements taken with an array of diodes in water and radiographic film in polystyrene.
Table II contains absolute dose values measured at single points for the seven $15 \mathrm{MV}$ fields reported above. The dose was measured at a high dose, low gradient area. The relative measurements of the profiles taken with diode array and film were normalized at these points. The agreement between calculations and measurements was better than $\pm 3 \%$. Table III contains a series of absolute dose measurements over a couple of dose profiles reported in Fig. 3 (cases 1 and 4). The agreement is around $0.8 \%$ and $2.6 \%$, respectively.

Figure 4 shows the gamma index criterion for two typical cases. The gamma calculation was done with $3 \%$ tolerance for dose, $3 \mathrm{~mm}$ for distance. In both cases, most of the image has a gamma lower than 1 , the points above 1.5 being very few. Typical values of the number of points with $\gamma>1$ are of the order of $\sim 1 \%$, and with $\gamma>1.5$ are less than $1 \%$. The averaged gamma value for all fields except case 4 is between 0.2 and 0.3 and for case 4 is 0.6 . The discrepancies between the measured and calculated doses appear because the fluence cannot be exactly determined in the segment edges. Regions of $\gamma>1$ in Fig. 4 (black areas) are related to this fact.

Figure 5 shows the error histograms according to the gamma criterion for all the seven cases studied. The gamma index is evaluated in the $200 \times 200$ points central square. This square completely encompasses the radiation field dimensions for all cases presented. This final test gives great confidence on the dose calculations in IMRT situations with our pencil beam kernels and the calculation formalism employed. Improving the deconvolution procedure to take into account dose contribution at isocenter from secondary radiation originated at higher radii would still improve the calculations and consequently the agreement with the measurements and the gamma index test.

Cases 4 and 6 show the highest discrepancies between calculated and measured data. These two cases have the largest segments, as can be seen in Table I. The modulation is not as high as in other situations presented and that makes the kernel tail influence more important. The discrepancies are related to the limitation in the information from high radii and this is expected to be solved with an improved implementation of our procedure. The case of field 4 is spe-

TABLE III. Two series of absolute dose measurements over a line dose profile, for cases 1 and 4 (15 MV photons).

\begin{tabular}{|c|c|c|c|c|c|c|c|c|}
\hline \multirow[b]{2}{*}{ Point } & \multicolumn{4}{|c|}{ Case 1} & \multicolumn{4}{|c|}{ Case 4} \\
\hline & $\begin{array}{c}\text { Position } \\
\text { (mm) }\end{array}$ & $\begin{array}{c}\text { Calculated } \\
\text { dose } \\
(\mathrm{cGy})\end{array}$ & $\begin{array}{c}\text { Measured } \\
\text { dose } \\
(\mathrm{cGy})\end{array}$ & $\begin{array}{c}\text { Difference } \\
(\%)\end{array}$ & $\begin{array}{c}\text { Position } \\
\text { (mm) }\end{array}$ & $\begin{array}{c}\text { Calculated } \\
\text { dose } \\
(\mathrm{cGy})\end{array}$ & $\begin{array}{c}\text { Measured } \\
\text { dose } \\
(\mathrm{cGy})\end{array}$ & $\begin{array}{l}\text { Difference } \\
(\%)\end{array}$ \\
\hline 1 & Center & 24.1 & 24.0 & 0.4 & Center & 25.9 & 26.8 & -3.4 \\
\hline 2 & +30 & 40.0 & 39.7 & 0.8 & +20 & 31.8 & 32.6 & -2.5 \\
\hline 3 & -30 & 24.5 & 24.5 & 0.0 & -20 & 32.7 & 33.4 & -2.1 \\
\hline 4 & +60 & 40.3 & 40.7 & -1.0 & +50 & 40.7 & 41.7 & -2.4 \\
\hline 5 & -60 & 38.4 & 39.2 & -2.0 & -50 & 41.9 & 43.0 & -2.6 \\
\hline 6 & & & & & +70 & 39.8 & 40.6 & -2.0 \\
\hline 7 & & & & & -70 & 40.3 & 41.7 & -3.4 \\
\hline Mean absolute difference & & & & 0.8 & & & & 2.6 \\
\hline
\end{tabular}



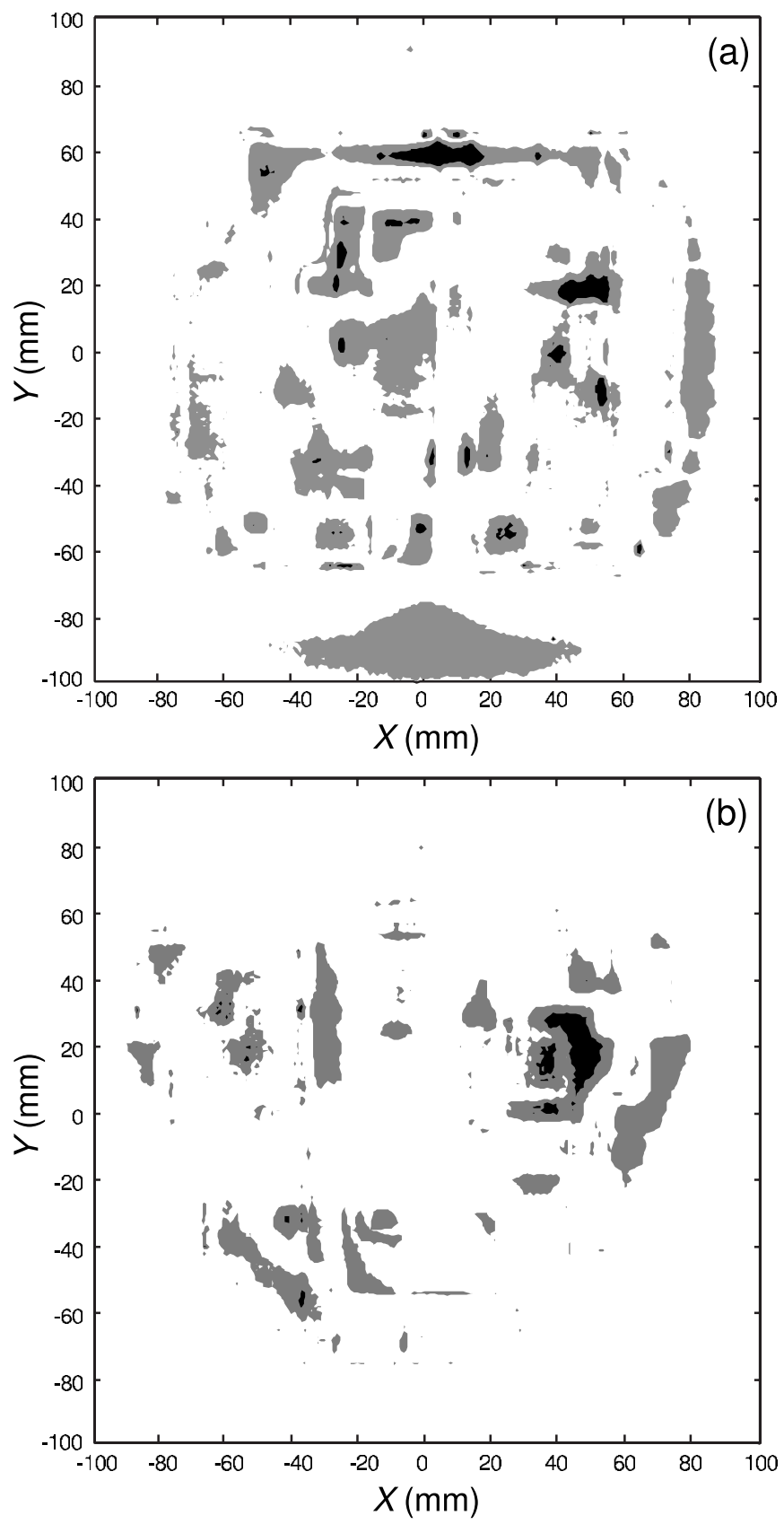

FIG. 4. Gamma index for (a) case 1 and (b) case 7 reported. The gamma index is evaluated in the $200 \times 200$ point central square of Fig. $3 \mathrm{~b}$ (case 1) and Fig. 3n (case 7). White color indicates a gamma value below 0.5 , gray between 0.5 and 1 , and black above 1 . The big gray zones [for example, in part (a), $-100<y<-80,-40<x<40]$ correspond to out of field areas, where dose is low.

cial since it has the largest segments. For case 4, the biggest segment is $224 \mathrm{~cm}^{2}$ and the mean area is $113.3 \mathrm{~cm}^{2}$. Consequently, the obtained gamma values tend to be high $(\gamma$ $>1,16 \%$ of the points; $\gamma>1.5,1 \%$ ).

The agreement in dose distributions is very good. Thus, kernels obtained with 2D deconvolution can be used for IMRT dose calculations with accuracy, in particular as a double-check of the calculations provided by commercial treatment planning systems on homogeneous media.

\section{CONCLUSION}

The main purpose intended in this study was to develop a deconvolution technique useful for obtaining pencil beam kernels that lead to accurate dose calculations in IMRT conditions. Moreover, a calculation formalism for IMRT is provided, as well as a complete set of measurements of absorbed dose in IMRT fields.

The pencil beam kernel derivation procedure presents some advantages over the initial work by Chui and Mohan. The former uses a pure 2D deconvolution technique that allows the derivation of a $2 \mathrm{D}$ kernel, as required by the pencil beam approach. In IMRT dose plans, the dose calculation in the areas where there exists superposition of penumbras or lack of electronic equilibrium, as is the case in the corners of the segments, can be very difficult. With a 2D kernel these effects can be taken into account properly. As the pencil beam kernels derived have the physically required shape and revolution symmetry, their convolution with the IMRT fluence maps leads to accurate dose calculations $(<3 \%)$ for treatment times (monitor units) as well as for dose distributions, represented in dose profiles and gamma index maps. This technique can be potentially used to derive pencil beam kernels for other types of radiation, such as electron, proton, and carbon ion beams.

The motivation for deriving these kernels, which is an important application, is to double-check the TPS calculations in IMRT dose plans. The pencil beams obtained are completely independent of those employed by commercial TPS, since the latter are usually calculated by Monte Carlo simulations. As has been shown, the results given in these calculations are highly accurate.

\section{ACKNOWLEDGMENTS}

The authors thank Dr. Chen-Shou Chui (Sun-Yat Sen Cancer Center, Taipei, Taiwan) and Dr. Didier Lemoine (Université Paul Sabatier, Toulouse Cedex, France) for fruitful scientific discussions. The authors also acknowledge the anonymous Associate Editor and Referee because of their careful and constructive criticism during the review of the manuscript.

\section{APPENDIX: THE DISCRETE ZEROTH-ORDER HANKEL TRANSFORM}

The calculation of integral (9) implies a full knowledge of the function $f(r)$. But actually, in most real problems we only have access to a discrete set of values $f\left(r_{i}\right)$, where the positions $r_{i}$ can or cannot be equally spaced. In the Fourier transform, there is a discrete version similar to the Fourier series that can be used to analyze these discrete data. The idea is to obtain a similar tool in the case of the Hankel transform. In the Fourier case, the periodicity of the signal allows the restriction to the first period. The main problem is that the analogous Hankel discrete transform implies the use of infinite series in the radial direction. In practice, we do not have an infinite amount of data for calculating the discrete Hankel transform. Thus, we need to achieve a compromise between 

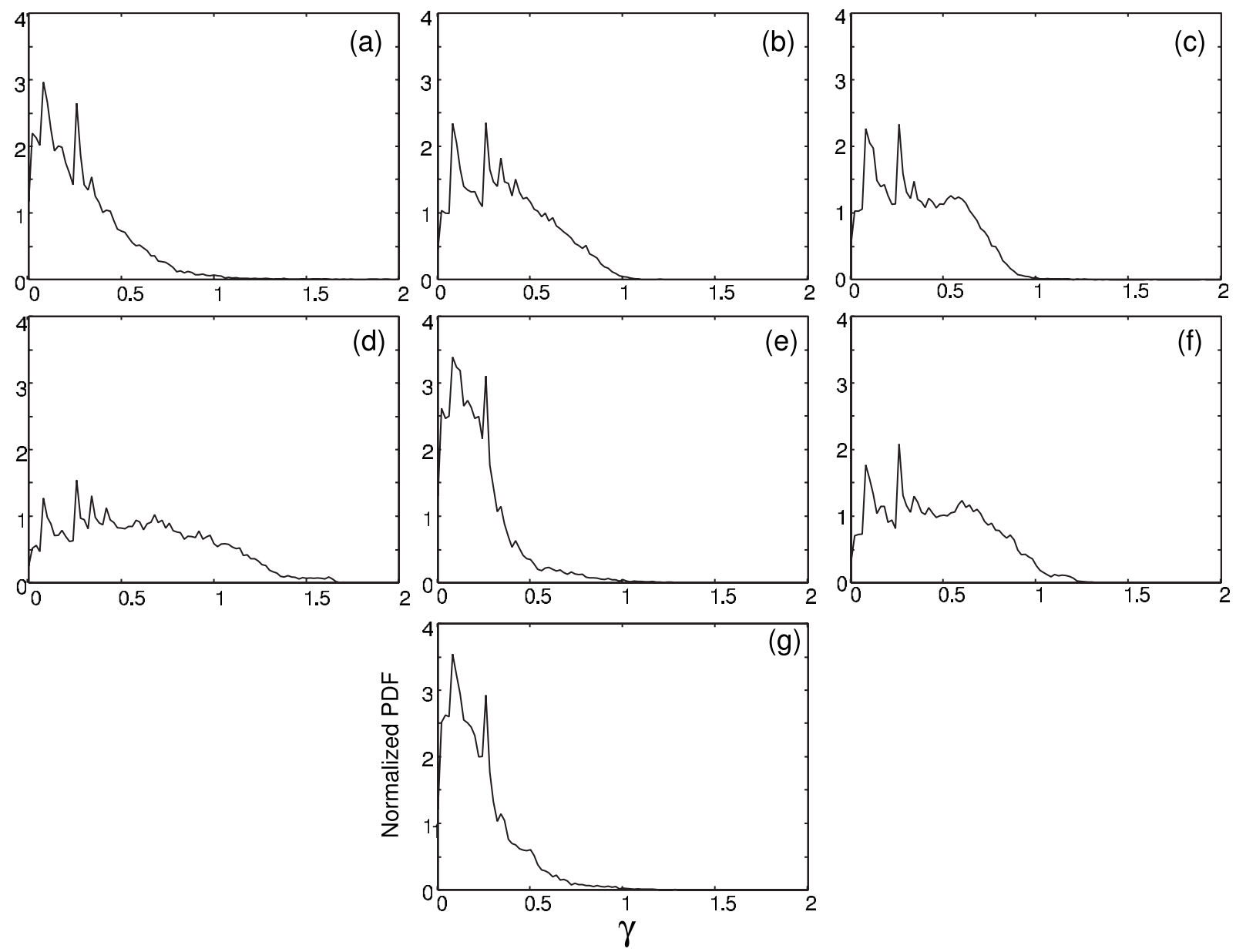

Fig. 5. Error histograms according to the gamma index criterion for the seven cases studied. The gamma index is evaluated in the $200 \times 200$ point central square of Figs. 3(b), 3(d), 3(f), 3(h), 3(j), 3(1), and 3(n).

accuracy and amount of data. The quasi-discrete version of the Hankel transform is defined as follows (both direct and back transforms):

$$
\begin{aligned}
& F\left(q_{m}\right)=\frac{1}{\pi Q^{2}} \sum_{n=1}^{N} f\left(r_{n}\right) J_{0}\left(2 \pi r_{n} q_{m}\right) J_{1}^{-2}\left(j_{n}\right), \\
& f\left(r_{n}\right)=\frac{1}{\pi R^{2}} \sum_{m=1}^{N} F\left(q_{m}\right) J_{0}\left(2 \pi r_{n} q_{m}\right) J_{1}^{-2}\left(j_{m}\right),
\end{aligned}
$$

where $j_{i}$ are the $i$ th zeros of the first kind zeroth-order Bessel function $J_{0}, r_{n}=\left(j_{n} / 2 \pi Q\right)$, and $q_{m}=\left(j_{m} / 2 \pi R\right)$. The data extend up to $r=R$ and $q=Q$, i.e., $f(r>R)=0$ and $F(q>Q)=0$, and the relationship between the number of data $N$ and the maximum radius in real and conjugated spaces $R$ and $Q$ is $j_{N+1}=2 \pi R Q$.

The accuracy of the zeroth-order discrete Hankel transform depends on the number of data available $N$. We can see from the definition that the zeroth-order Hankel transform is self-inverse, and that variables $r$ and $q$, as well as $f$ and $F$, are symmetric.

The factors $\left(J_{1}\left(j_{m}\right) R\right)^{-2}$ and $\left(J_{1}\left(j_{n}\right) Q\right)^{-2}$ in Eqs. (A1) and (A2) appear to verify the following orthogonality condition (on an interval $[0, S], S$ can take the value $R$ or $Q$ ):

$$
\int_{0}^{S} J_{0}\left(j_{n} \frac{s}{S}\right) J_{0}\left(j_{m} \frac{s}{S}\right) s d s=\frac{1}{2} S^{2} J_{1}^{2}\left(j_{n}\right) \delta_{m, n}
$$

where $j_{m}$ is again the $m$ th zero for the function $J_{0}$, and $\delta_{m, n}$ is the Kronecker delta.

In the discrete case of the transform, we need the components of the functions base to be orthogonal. We achieve this in the Fourier case by setting the data equally spaced. In this case, only certain discrete frequencies form the sines and cosines base. According to Eq. (A3), the data in the zerothorder Hankel transform should be spaced according to the zeros of the first kind of order zero Bessel function $J_{0}$.

a) Telephone: 34948255400 x4913; Fax: 349482555 00; Electronic mail: jazcona@unav.es

${ }^{1}$ A. L. Boyer, E. B. Butler, T. A. DiPetrillo, M. J. Engler, B. Fraas, W. Grant III, C. C. Ling, D. A. Low, T. R. Mackie, R. Mohan, J. A. Purdy, M. Roach, J. G. Rosenman, L. J. Verhey, J. W. Wong, R. L. Cumberlin, H. Stone, and J. R. Palta, "Intensity-modulated radiotherapy: Current status and issues of interest," Int. J. Radiat. Oncol. Biol. Phys. 51, 880-914 (2001).

${ }^{2}$ G. A. Ezzell, J. M. Galvin, D. Low, J. R. Palta, I. Rosen, M. B. Sharpe, P. Xia, Y. Xiao, L. Xing, and C. X. Yu, "Guidance document on delivery, treatment planning, and clinical implementation of IMRT: Report of the IMRT subcommittee of the AAPM radiation therapy committee," Med. Phys. 30, 2089-2115 (2003).

${ }^{3}$ J. M. Galvin, G. Ezzell, A. Eisbrauch, C. Yu, B. Butler, Y. Xiao, I. Rosen, 
J. Rosenman, M. Sharpe, L. Xing, P. Xia, T. Lomax, D. A. Low, and J. Palta, "Implementing IMRT in clinical practice: A joint document of the American Society for Therapeutic Radiology and the American Association of Physicists in Medicine," Int. J. Radiat. Oncol. Biol. Phys. 58, 1616-1634 (2004).

${ }^{4}$ J. H. Kung, G. T. Y. Chen, and F. K. Kuchnir, "A monitor unit verification calculation in intensity modulated radiotherapy as a dosimetry quality assurance," Med. Phys. 27, 2226-2230 (2000).

${ }^{5}$ L. Xing, Y. Chen, G. Luxton, J. G. Li, and A. L. Boyer, "Monitor unit calculation for an intensity modulated photon field by a simple scattersummation algorithm," Phys. Med. Biol. 45, N1-N7 (2000).

${ }^{6}$ Y. Watanabe, "Point dose calculations using an analytical pencil beam kernel for IMRT plan checking," Phys. Med. Biol. 46, 1031-1038 (2001). ${ }^{7}$ Y. Yang, L. Xing, J. G. Li, J. Palta, Y. Chen, G. Luxton, and A. L. Boyer, "Independent dosimetric calculation with inclusion of head scatter and MLC transmission for IMRT," Med. Phys. 30, 2937-2947 (2003).

${ }^{8}$ C. Chui, T. LoSasso, and A. Palm, in A Practical Guide to Intensity Modulated Radiation Therapy, Memorial Sloan-Kettering Cancer Center, edited by Z. Fuks, S. A. Leibel, and C. C. Ling (Medical Physics, Madison, 2003).

${ }^{9}$ C. P. Ceberg, B. E. Bjärngard, and T. C. Zhu, "Experimental determination of the dose kernel in high-energy x-ray beams," Med. Phys. 23, 505-511 (1996).

${ }^{10}$ Y. Yang, L. Xing, A. L. Boyer, Y. Song, and Y. Hu, "A three-source model for the calculation of head scatter factors," Med. Phys. 29, 20242033 (2002).

${ }^{11}$ R. Mohan and C. Chui, "Use of fast Fourier transforms in calculating dose distributions for irregularly shaped fields for three-dimensional treatment planning," Med. Phys. 14, 70-77 (1987).

${ }^{12}$ A. Ahnesjö, M. Saxner, and A. Trepp, "A pencil beam model for photon dose calculations," Med. Phys. 19, 263-273 (1992).
${ }^{13}$ T. Bortfeld, W. Schlegel, and B. Rhein, "Decomposition of pencil beam kernels for fast dose calculations in three-dimensional treatment planning," Med. Phys. 20, 311-318 (1993).

${ }^{14}$ J. D. Bourland and E. L. Chaney, "A finite-size pencil beam model for photon dose calculations in three dimensions," Med. Phys. 19, 1401-1412 (1992).

${ }^{15}$ B. E. Bjärngard and H. Shackford, "Attenuation in high energy x-ray beams," Med. Phys. 21, 1069-1073 (1994).

${ }^{16}$ B. E. Bjärngard and P. Vadash, "Analysis of central-axis doses for highenergy x rays," Med. Phys. 22, 1191-1195 (1995).

${ }^{17}$ B. E. Bjärngard, P. Vadash, and T. Zhu, "Doses near the surface in high energy X-ray beams," Med. Phys. 22, 465-468 (1995).

${ }^{18}$ P. Storchi and E. Woudstra, "Calculation of the absorbed dose distribution due to irregularly shaped photon beams using pencil beam kernels derived from basic beam data," Phys. Med. Biol. 41, 637-656 (1996).

${ }^{19}$ P. R. M. Storchi, L. J. Van Battum, and E. Woudstra, "Calculation of a pencil beam kernel from measured photon beam data," Phys. Med. Biol. 44, 2917-2928 (1999).

${ }^{20} \mathrm{C}$. S. Chui and R. Mohan, "Extraction of pencil beam kernels by the deconvolution method," Med. Phys. 15, 138-144 (1988).

${ }^{21}$ R. N. Bracewell, The Fourier Transform and its Applications, 3rd ed. (McGraw-Hill, New York, 2000).

${ }^{22}$ D. Lemoine, "The discrete Bessel transform algorithm," J. Chem. Phys. 101, 3936-3944 (1994).

${ }^{23}$ D. Lemoine, "A note on orthogonal discrete Bessel representations," J. Chem. Phys. 118, 6697-6699 (2003).

${ }^{24}$ L. Yu, M. C. Huang, M. Z. Chen, W. Z. Chen, W. D. Huang, and Z. Z. Zhu, "Quasi-discrete Hankel transform," Opt. Lett. 23, 409-411 (1998).

${ }^{25}$ D. A. Low, W. B. Harms, S. Mutic, and J. A. Purdy, "A technique for the quantitative evaluation of dose distributions," Med. Phys. 25, 656-661 (1998). 